\title{
Impacto da desnutrição na qualidade de vida de idosos institucionalizados no município de Itabuna - BA
}

\section{Impact of malnutrition on the quality of life of institutionalized elderly in the municipality of Itabuna - BA}

\section{Impacto de la desnutrición en la calidad de vida de ancianos institucionalizados en el municipio de Itabuna - BA}

\author{
Rodolfo Paula Vieira iD \\ Caliana R. Lima ${ }^{1}$ \\ 1 calianarochalima@hotmail.com \\ Endereço para correspondência: \\ Caliana R. Lima \\ Praça Guilherme Xavier, 35 \\ Banco da Vitória \\ 45661-236 - Ilhéus - Bahia - Brasil \\ E-mail: calianarochalima@hotmail.com
}

Recebido: 02.09.2020

Modificado: 03.09.2020

Aceito: 11.09.2020

\section{RESUMO}

A senescência, apesar de ser um processo natural, submete o organismo a diversas alterações anatômicas e funcionais, com repercussões nas condições de saúde e nutricional do idoso. A procura de instituições de longa permanência para idoso (ILPI), diante da perspectiva demográfica e social, está aumentando e representando uma nova alternativa de moradia para estes indivíduos. A institucionalização pode trazer inúmeras consequências, tanto positivas quanto negativas, como reflexos da adaptação à vida asilar. O objetivo do estudo foi de analisar a prevalência de desnutrição em idosos residentes em instituições geriátricas na cidade de Itabuna - BA correlacionando fatores psicológicos, cognitivos e a deficiência da capacidade funcional. Trata-se de um estudo qualitativo e quantitativo, com idosos integrantes de uma ILPI do município de Itabuna - BA, sendo composta por indivíduos de ambos os sexos com idade maior ou igual a 60 anos, que aceitaram participar do estudo e em condições de realização da avaliação antropométrica. A amostra parcial foi composta por 43 idosos e os dados antropométricos coletados foram: peso, altura, IMC, circunferência do braço e da panturrilha e dados dietéticos foram obtidos através do questionário da Mini Avaliação Nutricional (MAN). As características das ILPI podem contribuir para o perfil nutricional encontrado. A obtenção de indicadores estatísticos de porcentagem de idosos com risco nutricional institucionalizados e estudos analíticos possibilitam a realização de intervenções precoces que favoreçam uma melhoria na qualidade de vida destes indivíduos que apresentem riscos nutricionais.

PALAVRAS-CHAVE: Envelhecimento. Nutrição do idoso. Desnutrição.

Aging, despite being a natural process, submits the organism to several anatomical and functional changes, with repercussions 
on the health and nutrition conditions of the elderly. The demand for long-stay institutions for the elderly (ILPI), given the demographic and social perspective, is increasing and representing a new housing alternative for these individuals. Institutionalization can bring countless consequences, both positive and negative, as reflections of adaptation to asylum life. The objective of this study is to analyze the prevalence of malnutrition in elderly residents of geriatric institutions in the city of Itabuna - BA, correlating psychological, cognitive and functional capacity deficiencies. This is a qualitative and quantitative study with a sample that should be composed with members of an ILPI of the municipality of Itabuna - BA, being composed of individuals of both sexes with age greater than or equal to 60 years, who accepted to participate in the study and under conditions of anthropometric evaluation. The partial sample consisted of 43 elderly people and the anthropometric data collected were: weight, height, BMI, circumference of the arm and calf, and dietary data were obtained through the Mini Nutrition Assessment (MAN) questionnaire. The characteristics of ILPI may contribute to the nutritional profile found. The achievement of statistical indicators of percentage of elderly people with institutionalized nutritional risk and analytical studies make it possible to carry out early interventions that favor an improvement in the quality of life of these individuals who present nutritional risks.

KEYWORDS: Aging. Elderly nutrition. Malnutrition.

\section{RESUMEN}

La senescencia, a pesar de ser un proceso natural, somete al organismo a varios cambios anatómicos y funcionales, con repercusiones en las condiciones de salud y nutrición de los ancianos. La demanda de centros de atención de larga duración para personas mayores (ILPI), en vista de la perspectiva demográfica y social, está aumentando y representa una nueva alternativa de vivienda para estas personas. La institucionalización puede tener numerosas consecuencias, tanto positivas como negativas, como reflejos de adaptación a la vida de asilo. El objetivo del estudio fue analizar la prevalencia de desnutrición en ancianos residentes en instituciones geriátricas de la ciudad de Itabuna - BA, correlacionando factores psicológicos, cognitivos y deficiencia de capacidad funcional. Se trata de un estudio cualitativo y cuantitativo, con ancianos integrantes de un LTCF del municipio de Itabuna - BA, conformado por individuos de ambos sexos de 60 años o más, quienes aceptaron participar del estudio y en condiciones evaluación antropométrica. La muestra parcial estuvo compuesta por 43 ancianos y los datos antropométricos recolectados fueron: peso, talla, IMC, circunferencia del brazo y pantorrilla y los datos dietéticos se obtuvieron mediante el cuestionario de la Mini Evaluación Nutricional (MAN). Las características de LTCI pueden contribuir al perfil nutricional encontrado. La obtención de indicadores estadísticos del porcentaje de ancianos con riesgo nutricional institucionalizado y estudios analíticos posibilitan intervenciones tempranas que favorezcan una mejora en la calidad de vida de estos individuos que presentan riesgos nutricionales.

PALABRAS CLAVE: Envejecimiento. Nutrición del anciano. Desnutrutrición. 


\section{INTRODUÇÃO}

Define-se como idoso todo indivíduo com idade igual ou superior a 60 anos para países em desenvolvimento ou 65 anos, no caso de nações desenvolvidas ${ }^{1}$.

Observa-se que desde o início da década de 60 vem ocorrendo alterações na estrutura etária da população brasileira, onde o número de pessoas idosas está aumentando mais rápido do que o número de crianças, o que ocasiona a expansão da expectativa de vida das pessoas².

A senescência, apesar de ser um processo natural, submete o organismo a diversas alterações anatômicas e funcionais, com repercussões nas condições de saúde e nutricional do idoso. Além dos condicionantes específicos desse estado fisiológico, existem outros fatores que podem afetar o estado nutricional dessa população, tais como: situação social (pobreza, isolamento social), alterações psicológicas (demência, depressão), condição de saúde (doenças crônicas, disfagia, polifarmácia, alterações na mastigação, perda da capacidade funcional e autonomia), entre outros ${ }^{3}$.

A compreensão sobre o grau de associação entre esses fatores pode favorecer a elaboração de propostas que visem à melhoria da saúde, do estado nutricional e da qualidade de vida destes sujeitos ${ }^{3}$. O ato ou efeito de envelhecer no Brasil, mostra grande complexidade, sendo considerado um problema de ordem social. A procura de instituições de longa permanência para idoso (ILPI), diante da perspectiva demográfica e social, está aumentando e representando uma nova alternativa de moradia para os anciões ${ }^{4}$.

A família é, ou pelo menos deve ser um suporte na proteção ao idoso fragilizado, entretanto têm-se verificado, principalmente nos grandes centros urbanos, modificações na estrutura familiar e na dinâmica da sociedade, com a inserção cada vez maior de integrantes da família ao mercado de trabalho, o que resulta no enfraquecimento do suporte de cuidado a estes indivíduos e no consequente aumento da institucionalizaçãos.

Diante das alterações peculiares ao envelhecimento, verifica-se que a avaliação do estado nutricional individualizada é essencial para diagnosticar riscos nutricionais precocemente, consequentemente, contribuir para proporcionar ao paciente idoso o restabelecimento de suas funções orgânicas, assim como melhorar sua qualidade de vida ${ }^{6}$.

A institucionalização pode trazer inúmeras consequências, tanto positivas quanto negativas, como reflexos da adaptação à vida asilar. A mudança para a instituição pode significar para o idoso uma espécie de confinamento, uma vez que esse se vê muitas vezes privado de suas atividades familiares e rotineiras ${ }^{3}$. No Brasil, o risco de morte por desnutrição proteica em idosos vem crescendo e está relacionado com problemas sociais e desigualdades no acesso à alimentação saudável. Além de estar relacionada com alterações fisiológicas do envelhecimento, a má nutrição está diretamente ligada a doenças que diminuem o apetite e reduzem o paladar ${ }^{6}$.

Vários métodos objetivos e subjetivos têm sido utilizados para avaliar o estado nutricional desta faixa etária. Dentre eles, destacam-se os dados antropométricos como índice de massa corporal (IMC), circunferência muscular do braço (CMB), circunferência do braço (CB), prega cutânea tricipital (PCT), circunferência da panturrilha (CP), Mini Avaliação Nutricional (MAN), que juntamente com inquérito alimentar, como o recordatório de 24h (R24h), são parâmetros importantes para o diagnóstico precoce do estado nutricional.

Aárea de estudo foi o município de Itabuna, Bahia. Um município brasileiro do sul do estado a cerca de $430 \mathrm{~km}$ da capital, é a quinta cidade mais populosa do estado. A cidade, em conjunto com o município vizinho de Ilhéus, forma uma aglomeração urbana classificada pelo IBGE como uma capital regional B, exercendo influência em mais de 40 municípios que juntos apresentam pouco mais de um milhão de habitantes. Segundo levantamento realizado pelo Programa das Nações Unidas para o Desenvolvimento, o município apresenta o terceiro melhor Índice de Desenvolvimento Humano do Estado da Bahia, ficando atrás somente da capital baiana, Salvador, e do município de Lauro de Freitas.

Segundo dados do Censo, do Instituto Brasileiro de Geografia e Estatística (IBGE) ${ }^{7}$, habitavam na cidade de Itabuna, em 2019, 213.233 pessoas e a Bahia deverá ser o segundo estado brasileiro a iniciar o processo de decréscimo populacional, a partir de 2035, depois apenas do Piauí, que deverá ver sua população começar a diminuir em 2032.

A redução da população baiana, a ser iniciada em 2035, deverá levar o estado a ter, em 2060, 13.825 .958 de moradores, $6.7 \%$ ou 986.659 pessoas menos do que se estima que tenha em 2018. No município, poucas ações do poder público estão alinhadas com as demandas que o envelhecimento da população apresenta.

Quanto à assistência e à moradia aos idosos que não recebem assistência familiar e são institucionalizados, Itabuna conta com apenas dois abrigos a Fundação Dr. Balduíno e o Lar São Francisco que atende em média 90 e 85 idosos respectivamente, necessitando assim, de medidas preventivas urgentes mais atentas ao envelhecimento populacional nesta região.

O objetivo do presente estudo foi de analisar a prevalência de desnutrição, em idosos residentes em instituições geriátricas na cidade de Itabuna - BA correlacionando fatores psicológicos, cognitivos e a deficiência da capacidade funcional com a presença ou ausência de risco nutricional para estes indivíduos. Favorecendo assim, a construção de um cenário estatístico que permita a análise e a construção de indicadores que possibilitem a obtenção de menores taxas de desnutrição e 
consequentemente maior independência nas Atividades de Vida Diária (AVD) na população idosa institucionalizada.

\section{REVISÃO DE LITERATURA}

\section{Envelhecimento Populacional}

A transição demográfica inicia com a redução das taxas de mortalidade e, depois de um tempo, com a queda das taxas de natalidade, provocando significativas alterações na estrutura etária da população. O Brasil, à semelhança de outros países, está vivenciando um processo de envelhecimento rápido e intenso, o que demanda ações integradas para atendimento adequado à realidade vivida por esta população ${ }^{8}$. Este fenômeno tem ocasionado uma inversão da pirâmide etária, atuando no processo de transformação da velhice em um drama social ${ }^{4}$.

O envelhecimento populacional traz consigo problemas de saúde que desafiam os sistemas de saúde e de previdência social. Envelhecer não significa necessariamente adoecer ${ }^{8}$. A menos que exista doença associada, o envelhecimento está associado a um bom nível de saúde. Segundo as projeções estatísticas da OMS, em 2025, a população brasileira, quintuplicará, enquanto que o grupo etário com idade superior a 60 anos será 15 vezes maior, representando 34 milhões de pessoas. Sendo assim, o Brasil ocupará o sexto lugar do mundo em contingente de idosos ${ }^{2}$.

Para o futuro próximo, espera-se um crescimento a taxas elevadas da população muito idosa (80 anos e mais), como resultado das altas taxas de natalidade observadas no passado recente e da continuação da redução da mortalidade nas idades avançadas. No entanto, a certeza do crescimento desse segmento populacional está sendo acompanhada pela incerteza das condições de cuidados que experimentarão os longevos, afirma ${ }^{9}$.

Diante desse contexto evidencia que, o objetivo dos gestores das áreas sociais e de saúde deixou de ser apenas prolongar a vida, mas também buscar estratégias que possibilitem um envelhecimento mais saudável e ativo, caracterizado pela interação multidimensional entre saúde física e mental, independência nas atividades de vida diária, integração social, suporte familiar e independência econômica ${ }^{10}$.

Os países têm buscado, cada vez mais, compreender o processo de envelhecimento populacional, procurando alternativas para manter seus cidadãos idosos socialmente e economicamente integrados e independentes. Isso porque a presença crescente de pessoas idosas na sociedade impõe o desafio de inserir o tema do envelhecimento populacional na formulação das políticas públicas e de implementar ações de prevenção e cuidado direcionados às suas necessidades, subsidiando a organização de uma rede com capacidade para ofertar serviços e ações no âmbito da proteção social ${ }^{8}$.
Espera-se que o contingente de idosos atinja a magnitude de aproximadamente 30,9 milhões de pessoas, no ano de 2020 , vindo a constituir $14 \%$ da população brasileira, ocupando, então, o sexto lugar na classificação mundial ${ }^{11}$.

Observando os dados da atual transição demográfica brasileira sob a ótica de gênero, constatamos um processo de feminização da velhice, ou seja, quanto mais a população envelhece, mais feminina ela se torna. Hoje, as mulheres representam $55.5 \%$ da população idosa brasileira e $61 \%$ do contingente de idosos acima de 80 anos $^{7}$.

Essa representação feminina resulta da maior expectativa de vida das mulheres que, em média, vivem 8 anos a mais que os homens. Dentre os fatores que concorrem para esse fenômeno, especialistas destacam as mortes violentas (assassinatos e acidentes), cujas vítimas, quando jovens e adultas, são homens em mais de $90 \%$ dos casos e o acompanhamento médico contínuo maior entre as mulheres do que entre os homens ao longo de suas vidas ${ }^{12}$.

Acontece que, hoje, as famílias, sobretudo as mulheres, carecem de apoio institucional para oferecerem condições favoráveis para que os/às idosos/as tenham uma assistência à altura do merecido. E, provavelmente, essa situação tenderá a piorar enquanto o cuidado aos/às idosos/as não for considerado uma questão pública, de responsabilidade não apenas das famílias, mas também do Estado e da sociedade como um todo ${ }^{13}$.

\section{Aspectos Fisiopatológicos Relacionados ao Envelhecimento}

O envelhecimento, apesar de ser um processo natural, submete o organismo a diversas alterações anatômicas e funcionais, com repercussões nas condições de saúde e nutrição do idoso ${ }^{14}$.

O processo natural do envelhecimento envolve inúmeras transformações biológicas inerentes aos organismos e que ocorrem de maneira gradativa e premida por necessidades evolutivas ${ }^{15}$.

Muitas dessas mudanças são progressivas, ocasionando efetivas reduções na capacidade funcional, alterações do paladar (pouca sensibilidade para gostos primários como sal e doce), alterações de processos metabólicos do organismo e modificação da composição corporal ${ }^{14}$.

O envelhecimento fisiológico compreende uma série de alterações nas funções orgânicas devido exclusivamente aos efeitos da idade avançada sobre o organismo, fazendo com que o mesmo perca a capacidade de manter o equilíbrio homeostático e que todas as funções fisiológicas gradualmente comecem a declinar ${ }^{16}$.

A velocidade deste declínio depende de diversos fatores, genéticos e epigenéticos, que determinarão a resposta do organismo aos estímulos.

Alterações fisiológicas próprias da idade podem levar a uma atrofia e fraqueza muscular maior, a sarcopenia. Ademais, alterações endócrinas, nutricionais, 
mitocondriais, genéticas e comportamentais, como o sedentarismo ou a reduzida atividade física, são determinantes para a distinção do grau de sarcopenia, que varia muito entre os idosos, sendo mais acentuada nos membros inferiores ${ }^{15}$.

Ainda segundo o autor: as causas para a sarcopenia são múltiplas, sendo as principais a disfunção mitocondrial, alterações endócrinas, distúrbios nutricionais, imobilidade, inatividade física e doenças neurodegenerativas. Quanto aos mecanismos hormonais associados à sarcopenia, podemos citar a ativação do sistema renina-angiotensina e o consequente aumento dos níveis de angiotensina II, implicados na atrofia muscular por levarem ao aumento dos níveis da E3 ligaseatrogina e, assim, à proteólise.

Os fatores que interferem no apetite e ingestão de alimentos incluem, a deficiência visual, que diminuem o reconhecimento e apreciação das cores e texturas dos alimentos. Além disso, a mudança para uma instituição impõe alterações na rotina alimentar dos idosos podendo acarretar diminuição na sua ingestão, fragilizando sua saúde ${ }^{17}$.

A estatura começa a diminuir a partir dos 40 anos cerca de $1 \mathrm{~cm}$ por década. Essa perda se deve à diminuição da coluna vertebral devido a alterações nos discos intervertebrais. O pico de massa óssea é alcançado entre 30 e 40 anos de idade, sendo maior nos homens do que nas mulheres. Alguns anos após esse pico começa a ocorrer perda progressivamente de massa óssea, que é de aproximadamente $3.3 \%$ ao ano em homens e de $1 \%$ ao ano nas mulheres ${ }^{16}$.

Entre as principais mudanças fisiológicas que interferem no estado nutricional do idoso encontra-se a diminuição do metabolismo basal, redistribuição da massa corporal, alterações no funcionamento digestivo, alterações na percepção sensorial e diminuição da sensibilidade à sede ${ }^{18}$.

Outra alteração é o decréscimo do débito cardíaco máximo, associado à idade, decorrente da frequência cardíaca máxima, pois esta diminui de 6 a 10 batimentos por minuto $(\mathrm{bpm})^{19}$. O débito cardíaco submáximo ou em repouso, no entanto, é pouco influenciado pela idade. Porém o débito cardíaco máximo reduz-se progressivamente com o passar dos anos ${ }^{20}$.

Com o envelhecimento, o sistema nervoso apresenta alterações com redução no número de neurônios, redução na velocidade de condução nervosa, redução da intensidade dos reflexos, restrição das respostas motoras, do poder de reações e da capacidade de coordenações ${ }^{21}$.

No período compreendido entre os 20 e 90 anos, o córtex cerebral experimenta perda de $10 \%$ a $20 \%$ de massa, podendo ocorrer em outras partes do cérebro prejuízo de até $50 \%$. Assim, à medida que o cérebro envelhece, a atividade bioquímica (neurotransmissores) é afetada frequentemente ${ }^{22}$.

Esses autores indicam que, com o envelhecimento, o cérebro é passível de hipóxia (quantidade inadequada de oxigênio). Assim, com o envelhecimento, alterações na estrutura do sistema circulatório e na inatividade física, acarretam declínio na circulação sanguínea que conduz o oxigênio.

O envelhecimento normal reúne um declínio gradual nas funções cognitivas ${ }^{23}$. A capacidade intelectual do indivíduo idoso pode ser mantida sem dano cerebral até os 80 anos. No entanto, dificuldades de aprendizagens e esquecimento sem importância podem ser incluídos, juntamente com algumas alterações subtis que normalmente ocorrem em idosos com idade até 70 anos $^{24}$.

$\mathrm{O}$ ritmo de aprendizado torna-se mais lento em uma pessoa idosa e uma abordagem mais simples leva a uma redução no aprendizado dos elementos periféricos de uma tarefa. E a extensão da perda funcional pode ser ilustrada por mensurações, tais com o desempenho de grandes mestres de xadrez, que comumente atingem o seu máximo por volta dos $35 \operatorname{anos}^{20}$.

O envelhecimento e suas alterações de saúde levam o idoso ao estreitamento da sua inserção social. As alterações físicas, como perdas sensoriais (déficit auditivo e visual), déficits cognitivos, problemas osteoarticulares, sequelas ou descontrole de doenças crônicas, são fatores que limitam a mobilidade e a independência do idoso, prejudicando sua sociabilidade, atividades diárias e bem-estar ${ }^{25}$.

As condições de vida e as oportunidades que os sujeitos desempenham ao longo da vida influenciam diretamente o envelhecimento saudável do idoso, pois, para este, velhice é fruto da trajetória social exercida pelo indivíduo desde o nascimento $^{26}$.

\section{Indicadores de Desnutrição no Idoso}

Entre os idosos, o aumento da prevalência de enfermidades é fator de risco, sendo necessário o conhecimento das doenças, do estado nutricional, das modificações corporais, psicológicas e sociais desse grupo etário $^{27}$. A desnutrição é o distúrbio nutricional mais prevalente em idosos, que os predispõem a adquirir enfermidades que acentuam as desordens nutricionais frequentes no envelhecimento. Cabe ressaltar, que as alterações nutricionais podem contribuir ou ainda, exacerbar doenças crônicas e agudas, acelerando o desenvolvimento de doenças degenerativas e dificultando o prognóstico desse grupo etário ${ }^{2}$.

O diagnóstico nutricional em idosos deve ser feita de maneira cautelosa já que existem vários fatores que dificultam a avaliação nutricional do idoso, tais como alterações fisiológicas da própria idade, alterações da composição corporal, que afetam os parâmetros de antropometria específicos para idosos, à presença de doenças (afetando, por exemplo, a memória).

A desnutrição é resultado da falta de ingestão de nutrientes necessários ao desenvolvimento das funções corpóreas. Em pacientes idosos, ela é comum, pois, com a 
idade avançada, o consumo alimentar diário diminui. Além disso, os alimentos consumidos são de baixas calorias, contribuindo para a deficiência nutricional e desnutrição ${ }^{28}$.

Alguns autores consideram que os sinais de desnutrição podem ser difíceis de distinguir daqueles resultantes do processo natural de envelhecimento, porém se essa condição mórbida não for detectada, pode contribuir para o agravamento de manifestações clínicas associadas à inúmeras doenças crônicas e aumento de mortalidade.

O uso de múltiplos medicamentos, situação bastante comum na população idosa, influencia na ingestão de alimentos, nos processos de digestão, absorção e utilização dos diversos nutrientes, podendo comprometer o estado de saúde e nutricional do idoso. Além disso, entre os fatores de risco associados à desnutrição, pode-se incluir a deterioração da saúde oral e da sensibilidade a gostos primários ${ }^{18}$.

A depressão também pode afetar o estado nutricional do idoso institucionalizado, necessitando, assim, observar se a falta de apetite tem relação com a solidão ou demais acometimentos acarretados pela idade ${ }^{6}$.

O desequilíbrio nutricional no idoso está relacionado positivamente ao aumento da morbimortalidade, à susceptibilidade a infecções e à redução da qualidade de vida ${ }^{29}$.

A desnutrição também se manifesta como um dos resultados de uma variedade de condições, incluindo-se anemia, úlcera de pressão, fraturas ósseas, fragilidade, déficit cognitivo, desidratação, hipotensão ortostática e disfunção imune, as quais demonstram uma estreita relação com o estado nutricional dos idosos ${ }^{30}$.

Ainda segundo os autores, segundo estimativas feitas no início da década passada, o Brasil apresentava uma proporção de baixo peso entre homens idosos em torno de $20.7 \%$, e de $17 \%$ para as mulheres na mesma faixa etária. Em números absolutos, o país tinha, nesse período, cerca de 1.300.000 idosos com baixo peso ${ }^{17}$. Os dados atuais apontam para uma prevalência de desnutrição em idosos residentes em domicílios em nível de 1\% a 15\%, para idosos internados em hospitais essa proporção oscila entre 35\% e 65\% e para os idosos institucionalizados detecta-se desnutrição em $25 \%$ a $60 \%$. Em comparação com outros países, no Brasil o risco de morrer de desnutrição na velhice é $71 \%$ maior do que nos EUAe $32.13 \%$ maior do que na Costa Rica.

A intervenção nutricional em Geriatria deve ser orientada de maneira individual, em função das múltiplas alterações fisiológicas e doenças acometidas com o envelhecimento.

Nesta área, a avaliação do estado nutricional é considerada complexa devido às alterações naturais do envelhecimento. Desta forma, a particularidade de cada idoso também deve ser considerada, pois diversos aspectos, tais como idade, fatores biológicos e fatores fisiológicos podem mascarar os resultados ${ }^{2}$.

Tendo em vista a gama de problemas decorrentes de uma má nutrição, torna-se necessária a utilização de instrumentos para identificar essa complicação alimentar. Com isso, a mini-avaliação nutricional (MAN) tem se apresentado como um método não invasivo, de fácil aplicabilidade e rápida execução que ajuda no diagnóstico de desnutrição ou risco do aparecimento da mesma ${ }^{6}$.

O rastreamento do risco nutricional nos idosos é importante para detectar a necessidade de uma intervenção dietética, a fim de prevenir a presença de comorbidades, que interferem na expectativa e qualidade de vida destes indivíduos com idade avançada ${ }^{31}$.

Recomenda-se que seja estabelecida rotinas de triagem nutricional, facilitando e agilizando a identificação do paciente que necessita de intervenção nutricional. Para isso, faz-se necessária a utilização de métodos com indicadores que avaliem de forma correta e sistemática a gravidade do indivíduo ${ }^{32}$.

\section{Instituições de Longa Permanência para Idosos (ILPI)}

Para a Anvisa, ILPI são instituições governamentais ou não-governamentais, de caráter residencial, destinadas a domicílio coletivo de pessoas com idade igual ou superior a 60 anos, com ou sem suporte familiar, em condição de liberdade, dignidade e cidadania ${ }^{33}$.

É comum associar ILPI a instituições de saúde. Mas elas não são estabelecimentos voltados à clínica ou à terapêutica, apesar de os residentes receberem - além de moradia, alimentação e vestuário - serviços médicos e medicamentos ${ }^{9}$.

A Sociedade Brasileira de Geriatria e Gerontologia (SBGG), somente em 2003 definiu esses tipos de instituições, como Instituições de Longa Permanência para Idosos (ILPI). No manual de funcionamento para as ILPI, as define como "estabelecimento para atendimento integral institucional, cujo público alvo são pessoas com 60 anos ou mais, dependentes ou independentes, que não dispõem de permanecer com a família ou em seu domicílio" ${ }^{34}$.

A prevalência de idosos em Instituições de Longa Permanência (ILP) chega a $11 \%$ nos países em desenvolvimento e, no Brasil, esse percentual atinge 1.5\%, o que demonstra a importância dessas instituições como centros de promoção, proteção e reabilitação da saúde de idosos, devendo, portanto, prezar pela satisfação tanto de familiares quanto dos institucionalizados ${ }^{3}$.

Antes da existência dessas instituições, os idosos eram abrigados em ILPI de mendicidade, juntamente com os pobres, desempregados, crianças abandonadas e doentes mentais. Com o passar dos tempos, as demais categorias (pobres, desempregados, crianças abandonadas e doentes mentais) foram trabalhadas de modo isolado e no século XX o termo ILPI passou a ser uma instituição específica para abrigar idosos ${ }^{35}$.

A inadequação nutricional em Instituições de Longa Permanência para Idosos tem sido crescentemente reconhecida como um campo de pesquisas prioritário em todo o mundo. Estudos indicam que a desnutrição é um problema que atinge de 20 a $80 \%$ dos idosos em Instituições de Longa Permanência para Idosos (ILPIs), sendo o 
distúrbio nutricional mais importante observado em pessoas acima de $60 \operatorname{anos}^{36}$.

As dificuldades financeiras nas ILPIs filantrópicas podem deixar essas instituições em desvantagem no que se refere ao aspecto nutricional. Porém, a aceitação das refeições nas instituições de longa permanência para idosos poderá ser melhorada através do planejamento de cardápios que contemplem as preferências alimentares dos idosos e que atendam às suas necessidades nutricionais. Considerase, finalmente, a necessidade de atenção especial para os indivíduos que necessitam de alimentação assistida, possibilitando um estado nutricional equilibrado e uma melhor qualidade de vida para estes sujeitos ${ }^{37}$.

Muitas ILPIs continuam sendo desprovidas de profissionais qualificados, havendo falta de médicos, enfermeiros, nutricionistas e outros profissionais, sendo a maior parte realizada por cuidadores sem formação específica. No entanto de acordo com a portaria $\mathrm{n}^{\circ}$ 810/1989 e a SBGG, as ILPI devem contar com uma equipe multidisciplinar, além de outros trabalhadores para desempenho dos serviços gerais.

\section{MATERIAL E MÉTODOS}

\section{Da Coleta de Dados e Seleção da Amostra}

A amostra foi composta por 43 idosos, de ambos os sexos, moradores de uma Instituição Geriátrica no município de Itabuna - BA, após a autorização através do Termo de Consentimento Livre e Esclarecido (TCLE) Institucional e assinatura da carta de anuência para a autorização de utilização de informações concernentes a aplicação do questionário.

Os idosos que fizeram parte da amostragem desse estudo, foram submetidos a uma avaliação nutricional, que atenderam aos critérios de inclusão: idade igual ou superior a 60 anos, residentes da ILPI e em condições físicas e mentais para serem avaliados. Foram excluídos do estudo cadeirantes, acamados, com membros amputados e com déficit cognitivo.

Trata-se de um estudo quantitativo e qualitativo, aonde a coleta de dados foi realizada na instituição Fundação Dr. Balduíno Lopes, localizada no município de Itabuna - BA. A pesquisa foi realizada após a autorização expressa dos responsáveis pelo abrigo, tomando ciência dos objetivos do estudo.

Foi solicitado, a dispensa da assinatura do TCLE de cada componente da amostra, pois muitos idosos não possuíam condições cognitivas e funcionais para assinatura dos termos. Foram excluídos os acamados, com sérias limitações motoras, amputados e os com qualquer alteração congênita em membros inferiores que limitassem o deambular ou com alterações cognitivas que interferissem na aplicação do questionário da Mini Avaliação Nutricional (MAN).

O questionário utilizado com base na MAN analisou as seguintes variáveis: Mobilidade, Alterações Neuropsicológicas, Perda de Apetite, índice de Massa Corporal, Lesões de Pele, consumo de determinados grupos alimentares, alterações no apetite e perda de peso recente.

Os dados obtidos foram armazenados e, posteriormente, tabulados nos softwares Microsoft Office Excel no qual foram calculados as médias e porcentagens de cada idoso relacionando seu estado nutricional, com sua idade e sexo, respectivamente.

\section{Antropometria}

A avaliação do estado nutricional foi realizada por meio de dados antropométricos (peso, altura, Índice de Massa Corporal, Circunferência do Braço, e Circunferência da Panturrilha), Avaliação Dietética através do Recordatório 24 horas (R24h) e questionário da MAN. Cabe mencionar que a coleta dos dados também foi realizada por estudantes do curso técnico de Nutrição previamente treinados, visando minimizar distorções no diagnóstico final.

Foi realizada primeiramente as aferições antropométricas (peso, altura, CB e CP), cálculo de IMC e em seguida a aplicação do questionário da MAN.

Os idosos subiram na balança para a pesagem e para a medição de sua altura, sem chapéus ou adornos na cabeça. Estes continuaram em posição de Frankfort (arco orbital inferior alinhado em um plano horizontal com o pavilhão auricular), em posição ortostática, com a cabeça ereta, olhando para frente, com o corpo erguido com extensão máxima.

Após obtenção do peso e altura, foi determinado o IMC pela razão entre o peso atual e a altura ao quadrado $\left(\mathrm{Kg} / \mathrm{m}^{2}\right)$. Para os critérios de avaliação do IMC foi utilizado os pontos de corte, onde classificam-se os indivíduos idosos com IMC menor que $22 \mathrm{~kg} / \mathrm{m}^{2}$ magreza ou desnutrição, entre 22 e $27 \mathrm{~kg} / \mathrm{m}^{2}$ eutrofia e acima de $27 \mathrm{Kg} / \mathrm{m}^{2}$ sobrepeso.

As circunferências de braço $(\mathrm{CB})$ e panturrilha $(\mathrm{CP})$ serão aferidas com o auxílio de fita métrica e a análise dos dados indicativa de diagnóstico do estado nutricional dos idosos será realizada através da pontuação obtida no questionário da MAN, a qual indica a presença ou ausência de risco nutricional ao paciente. A CB e a CP são usadas como indicadores de massa muscular, sendo mensuradas em nível periférico.

$\mathrm{Na}$ aferição da CB, o idoso ficou de pé com a palma da mão voltada para coxa. Esta foi realizada com a fita métrica de aço plano, marca Sanny ${ }^{\circledR}$ colocada em torno do ponto médio do braço não dominante. A circunferência do braço é utilizada como indicador de reserva calórica e proteica.

$\mathrm{Na}$ aferição da CP, foi realizada na perna esquerda, com 
uma fita métrica inelástica, na sua parte mais protuberante, com o paciente com a perna dobrada formando um angulo de 90 graus com o joelho. De acordo com a OMS, a circunferência da panturrilha é aquela que fornece a medida mais sensível da massa muscular nos idosos. Esta medida indica alterações na massa magra que ocorrem com a idade e com o decréscimo na atividade física.

Segundo a Associação Brasileira de Nutrologia (ASBRAN,2015) é particularmente recomendada na avaliação nutricional de pacientes acamados. Deverá ser considerada adequada a circunferência igual ou superior a $31 \mathrm{~cm}$ para homens e mulheres e a circunferência braquial igual ou superior a $21 \mathrm{~cm}$.

\section{Mini Avaliação Nutricional (MAN)}

Um dos critérios utilizados para diagnóstico nutricional da amostra estudada também foi a aplicação do questionário MAN, que foi validado por diversos estudos, em diferentes populações.

O diagnóstico do estado nutricional demonstrou alta correlação com valores bioquímicos e antropométricos considerados padrão-ouro. O elevado custo de alguns exames bioquímicos na avaliação do estado nutricional possibilita que a MAN seja aplicada como medida prioritária, eficaz e de custo reduzido em idosos, objetivando rápida avaliação e intervenção. Todos os estudos revisados indicaram que a MAN apresenta grande especificidade em detectar precocemente o risco de desnutrição em idosos, o que é de grande valia no acompanhamento de idosos institucionalizados.

O questionário com base na MAN é composto por duas etapas, sendo a primeira composta por 4 questões sobre a avaliação antropométrica como redução de peso nos últimos 3 meses, IMC e circunferências do braço e panturrilha totalizando um escore máximo de 8 pontos, a segunda triagem contendo seis questões envolvendo quantidade de medicamentos utilizados por dia, estresse psicológico nos últimos três meses, assim como avaliação da mobilidade, problemas neuropsicológicos e lesões de pele totalizando um escore máximo de 8 pontos ,a terceira sobre avaliação dietética englobando perguntas sobre ingestão hídrica, modo de alimentar, quantidade de refeições feitas por dia, consumo e quantidade das porções de frutas ou vegetais por dia ,assim como de ovos, leguminosas, leite ou derivados e perda de apetite totalizando um escore de 10 pontos e a última sobre uma auto avaliação sobre a sua saúde em relação a outras pessoas da mesma idade e se acredita ter algum problema nutricional,com um total de 4 pontos. O escore total chega a um valor máximo de 30 pontos, onde escore com valores entre 17 e 23.5 pontos indica risco de desnutrição e escore com valor menor que de 17 pontos indica desnutrição.

No presente estudo, o tempo médio de aplicação do MAN foi de três minutos e também foi aplicado por estudantes do curso técnico de Nutrição, pois algumas questões necessitam de treinamento do profissional de saúde (como antropometria) ou implicam em julgamentos subjetivos, dessa forma é necessário que o profissional seja previamente treinado para sua aplicação não havendo dificuldades nesse quesito para a sua aplicação devido à sua simplicidade e praticidade.

\section{RESULTADOS}

A população total de residentes na instituição no período do estudo era de 88 idosos. Destes, 43 idosos (48.86\%) preencheram os critérios de inclusão e exclusão propostos pelo estudo. Sendo 27 do sexo feminino e 16 do sexo masculino com idades que variam entre 60 a 80 anos, dos quais $62.69 \%$ do sexo feminino e $37.20 \%$ do sexo masculino.

A idade média geral da amostra foi de $71.37 \pm(71.66$ anos para as mulheres e 70.87 para os homens), sendo o mais longevo com 80 anos.

A amostra parcial foi coletada na Fundação Dr. Balduíno Lopes, na cidade de Itabuna - BA, após a assinatura do Termo de Consentimento Livre e Esclarecido para a coleta de dados na Instituição. Métodos objetivos e subjetivos foram utilizados para avaliar o estado nutricional dos institucionalizados. O instrumento de coleta dos dados obtidos foi um questionário com base na Mini Avaliação Nutricional (MAN), aonde foi verificado dados antropométricos, dietéticos e uma avaliação da capacidade funcional. O IMC foi calculado com base nos dados de peso e altura obtidos, com o objetivo de avaliar o estado nutricional da população estudada. Este índice é calculado dividindo-se o peso pela altura ao quadrado. Os dados encontrados foram classificados de acordo com pontos de corte propostos por Lipschitz que preconiza, os indivíduos são classificados como baixo-peso com IMC $<22 \mathrm{~kg} / \mathrm{m}^{2}$; eutrofia, IMC entre $22 \mathrm{~kg} / \mathrm{m}^{2}$ e $27 \mathrm{~kg} / \mathrm{m}^{2}$; e acima do peso $\mathrm{IMC}>27 \mathrm{~kg} / \mathrm{m}^{2}$.

A análise do estado nutricional (EN) da amostra, adotando os critérios do IMC, demonstrou que $48.8 \%$ dos idosos estavam com baixo-peso, 27.9\% dos idosos eram eutróficos e $23.2 \%$ estavam com obesidade. A Tabela 1 apresenta a distribuição do EN segundo o IMC, categorizado segundo os três tipos de estado nutricional. 
Tabela 1 - Classificação do estado nutricional segundo o índice de massa corporal, em percentual.

\begin{tabular}{lll}
\hline IMC & Quantidade & $\%$ \\
\hline Desnutrido & 21 & $48.8 \%$ \\
Eutrofia & 12 & 27.95 \\
Acima do Peso & 10 & $23.2 \%$ \\
\hline Total & 43 & $100 \%$ \\
\hline
\end{tabular}

Nesta amostra parcial de idosos institucionalizados, encontrou-se uma prevalência de baixo-peso segundo o IMC de $48.8 \%$ na amostra geral $(n=43)$.

Ao analisar o estado nutricional nos três diferentes segmentos etários, o grupo etário que apresentou a maior porcentagem de desnutridos foram de 80 anos com 100\% com amostra de 4 idosos, no grupo de faixa etária de 70-79 foi de $35.7 \%$ para o sexo feminino, já no sexo masculino a faixa etária de 80 anos foi de $100 \%$ com 1 idoso somente, 70-79 anos, a porcentagem foi $62.5 \%$.

A diminuição do IMC com o avançar da idade pode ser atribuída à redução da massa muscular corporal e à diminuição da quantidade de gordura corporal, que tende a diminuir depois dos 70 anos. Desta forma, Lipschitz enfatiza que a dificuldade na utilização do IMC na avaliação do estado nutricional em idosos é agravado pela heterogeneidade que acompanha o envelhecimento e pela presença de doenças idade-dependentes. Sua utilização, porém, é importante tanto para a análise de indivíduos quanto para populações, permitindo comparação com estudos nacionais e internacionais, além de expressar as reservas energéticas do indivíduo.

Os resultados obtidos nesta pesquisa mostram que os pontos de corte estipulados por Lipschitz abrangem uma maior faixa de indivíduos com baixo-peso do que os pontos de corte

Os resultados obtidos que evidenciam a classificação do IMC segundo os gêneros, serão apresentados nas Figuras 1 e 2.

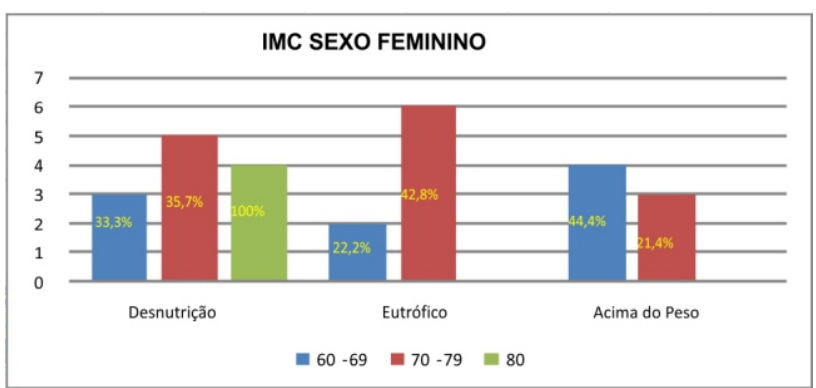

Figura 1 - Relação entre o estado nutricional e a idade, sexo feminino.

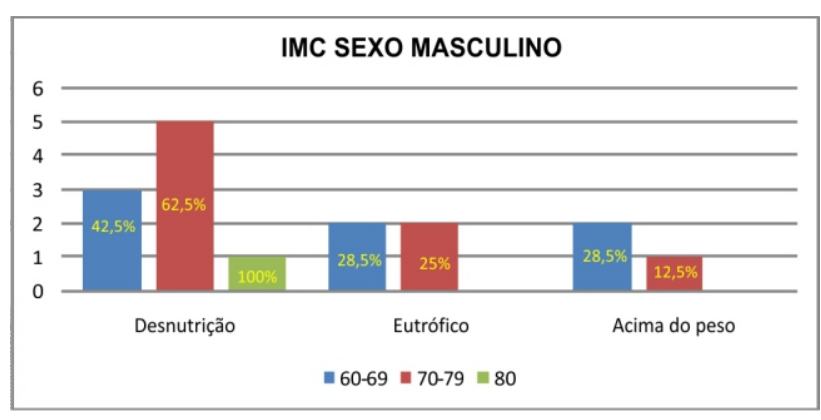

Figura 2 - Relação entre o estado nutricional e a idade, sexo masculino.

O IMC é o indicador antropométrico mais utilizado para avaliar o risco nutricional, por ser uma medida facilmente aplicável, não invasiva e de baixo custo. Em idosos, porém, seu emprego apresenta controvérsias em função do decréscimo de estatura, acúmulo de tecido adiposo, redução da massa corporal magra e diminuição da quantidade de água no organismo. Assim, vem sendo muito discutido o uso do IMC e dos limites de normalidade adotados para análise de desnutrição, sobrepeso e obesidade em idosos. Existem duas referências para a classificação do IMC: a primeira foi estipulada pela OMS que classifica indivíduos com IMC $<18.5 \mathrm{~kg} / \mathrm{m}^{2}$ como baixo-peso; eutrofia, IMC entre $18.5 \mathrm{~kg} / \mathrm{m}^{2}$ e $24.9 \mathrm{~kg} / \mathrm{m}^{2}$; sobrepeso, IMC entre $25 \mathrm{~kg} / \mathrm{m}^{2}$ e $29.9 \mathrm{~kg} / \mathrm{m}^{2}$; obesidade grau I, IMC entre $30 \mathrm{~kg} / \mathrm{m}^{2}$ e $34.9 \mathrm{~kg} / \mathrm{m}^{2}$; obesidade grau II, IMC entre $35 \mathrm{~kg} / \mathrm{m}^{2}$ e $39.9 \mathrm{~kg} / \mathrm{m}^{2}$; e obesidade grau III, IMC $>40 \mathrm{~kg} / \mathrm{m}^{2}$ e a outra, proposta por Lipschitz. Há críticas, no entanto, sobre o uso dos mesmos pontos de corte para classificar obesidade em adultos e idosos, pois mudanças na composição corporal, associadas ao processo de envelhecimento, devem ser consideradas. Já os pontos de corte propostos por Lipschitz levam em consideração as mudanças na composição corporal que ocorrem com o envelhecimento quando comparados com os indivíduos adultos por isso, se justifica a escolha do segundo método para aplicação do IMC.

A análise da classificação do EN obtido pela CB (Tabela 2) identificou diferenças significativas dentro da amostra, com proporções discrepantes em relação aos valores adotados como medidas para risco nutricional segundo a Mini Avaliação Nutricional (MAN), aonde valores inferiores a $21 \mathrm{~cm}$ é considerado risco de desnutrição, pois, identificou maior proporção de idosos com eutrofia do que em risco de desnutrição. Foram observadas diferenças significativas entre CB, CP e IMC entre as classificações do estado nutricional. Segundo a CB, porém, poucos idosos seriam identificados com desnutrição. Os dados obtidos serão demonstrados na Tabela 2. 
Tabela 2 - Distribuição da avaliação antropométrica segundo a circunferência do braço.

\begin{tabular}{lll}
\hline Circunferência do braço & Quantidade & Porcentagem \\
\hline $\mathrm{CB}>21$ & 13 & $30.23 \%$ \\
$21 \leq \mathrm{CB} \leq 22$ & 10 & $23.25 \%$ \\
$\mathrm{CB}>22$ & 20 & $46.51 \%$ \\
\hline Total & 43 & $100 \%$ \\
\hline
\end{tabular}

A classificação desta medida também pode ser pela distribuição em percentil, sendo considerados desnutridos aqueles idosos que se encontrarem no percentil igual ou abaixo de $5 \%$ e obesos aqueles que se encontrarem igual ou acima de $85 \%$.

Quando o estado nutricional foi analisado segundo a interpretação da $\mathrm{CP}$, a prevalência de desnutrição na amostra adotando os pontos de corte da MAN para risco de desnutrição, considerando adequada a circunferência igual ou superior a $31 \mathrm{~cm}$ para homens e para mulheres variou entre $62.7 \%$ e $37.2 \%$.

De acordo com a Organização Mundial de Saúde, a circunferência da panturrilha é aquela que fornece a medida mais sensível da massa muscular nos idosos. Esta medida indica alterações na massa magra que ocorrem com a idade e com o decréscimo na atividade física. A Tabela 3 apresenta essa distribuição.

Tabela 3 - Distribuição numérica da avaliação antropométrica segundo a circunferência da panturrilha.

\begin{tabular}{llc}
\hline Circunferência & Quantidade & $\%$ \\
\hline $\mathrm{CB}<31$ & 27 & $62.7 \%$ \\
$\mathrm{CP} \geq 31$ & 16 & $37.2 \%$ \\
\hline Total & 43 & 100
\end{tabular}

A MAN também pode avaliar o percentual de perda de peso nesta amostra estudada, conforme demonstrado na Tabela 4.

Durante o envelhecimento normalmente está ligada à falta de apetite, alteração do paladar ou devido a efeitos colaterais de remédios. Outro motivo comum é a demência, que faz com que a pessoa esqueça de fazer as refeições e de se alimentar de forma adequada.

Além do emagrecimento, também é normal ocorrer a perda de massa óssea, o que torna o idoso mais frágil e com maior risco de ter fraturas ósseas.
Tabela 4 - Distribuição numérica dos idosos segundo a perda de peso nos últimos 3 meses.

\begin{tabular}{llc}
\hline & Quantidade & $\%$ \\
\hline Não soube & 30 & 69.76 \\
Não perdeu peso & 03 & 6.97 \\
Superior a 3 quilos & 07 & 23.25 \\
\hline Total & 43 & 100 \\
\hline
\end{tabular}

Os resultados observados neste estudo mostraram que mais da metade dos idosos institucionalizados apresentaram risco nutricional ou desnutrição. Esses resultados evidenciam a necessidade de acompanhamento e terapia nutricional precoce e efetiva para essa população.

Estudos prévios têm confirmado a elevada prevalência de risco nutricional e desnutrição em idosos hospitalizados ou institucionalizados, justificando o estabelecimento de condutas e intervenções específicas que possam ser implementadas visando à melhora e/ou a não deterioração do estado nutricional, conforme evidenciado na Tabela 5 e Figuras 3 e 4 que demonstra a relação entre a idade, sexo e estado nutricional.

Apesar desse cenário se estender à faixa etária idosa, o risco nutricional nessa população aumenta proporcionalmente à idade. Aonde a faixa etária de 70 a 79 anos, em ambos os sexos apresentou uma maior prevalência segundo o escore total da Mini Avaliação Nutricional (MAN) um maior índice de desnutridos e risco de desnutrição, com escore total abaixo de 17 e entre 17 e 23 , respectivamente.

Tabela 5 - Classificação do estado nutricional segundo o escore total da MAN.

\begin{tabular}{lll}
\hline Escore: & Quantidade & $\%$ \\
\hline \multirow{2}{*}{ Bem Nutrido } & 15 & $34.9 \%$ \\
Risco de Desnutrição & 10 & $23.2 \%$ \\
Desnutrido & 18 & $41.9 \%$ \\
\hline Total & 43 & $100 \%$ \\
\hline
\end{tabular}

A população analisada constitui-se, na sua maior parte, por idosos do sexo feminino (62.7\%), assim como na população brasileira, de modo geral.

Além da influência da idade no risco nutricional e na desnutrição em idosos, os resultados observados demonstraram que pode haver uma influência do sexo, pode-se inferir que as mulheres, embora tendo perfil semelhante quanto à frequência de morbidades e idade apresentaram maior frequência de risco nutricional e desnutrição. 


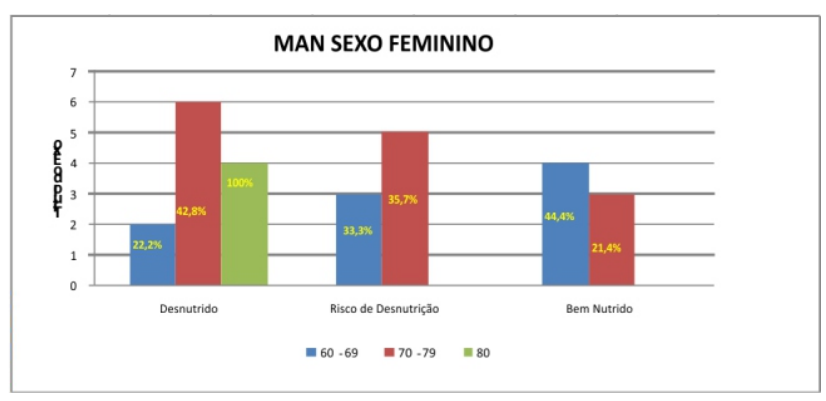

Figura 3 - Relação entre o estado nutricional e a idade, sexo feminino e masculino segundo a mini avaliação nutricional.

Ao analisarmos a figura podemos observar que no sexo feminino os desnutridos, a faixa etária que teve a maior porcentagem foi de 80 anos com $100 \%$ dos idosos, seguido pela faixa etária 70-79 que corresponde a $42.8 \%$, já no sexo masculino segue a mesma tendência, a faixa etária de 80 anos tem $100 \%$ de idosos desnutridos, vem logo atrás a faixa etária de 70-79 com 50\% dos desnutridos. Fica evidenciado neste estudo que quanto maior a idade maior o risco de desnutrição tanto para o sexo masculino quanto para o sexo feminino.

A elevada média de idade pode estar relacionada com o aumento da expectativa de vida, o que contribui para a maior incidência e agravamento de complicações das Doenças Crônicas Não Transmissíveis (DCNT), tornando mais frequentes as internações hospitalares e o tempo de hospitalização. Os resultados reforçam esse perfil ao identificarem que indivíduos na faixa etária de 80 anos tiveram maior risco nutricional e desnutrição.

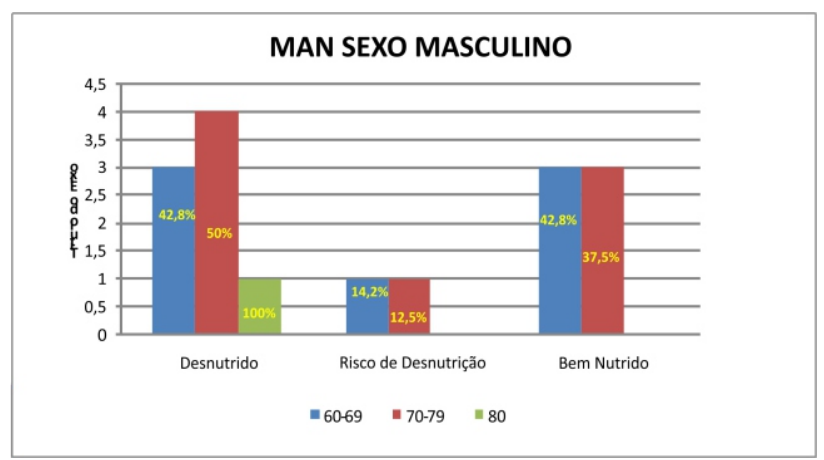

Figura 4 - Relação entre o estado nutricional e a idade, sexo masculino.

Entre aquilo que é servido nas ILPIs, muito embora o número mediano de refeições servidas ao dia (seis) e o número mínimo (quatro) não esteja inadequado, é preocupante que aILPI estudada não tenham oferecimento de alguma fruta no café da manhã. Idosos apresentaram ingestão abaixo do desejado de nutrientes importantes para a alimentação deles, como vitamina $\mathrm{C}$, folato, cálcio, potássio, zinco e fibras, os quais são normalmente encontrados em frutas e vegetais frescos.
A aplicação da MAN, também pode evidenciar um baixo consumo de leite e derivados, assim como de frutas e hortaliças. Esses resultados indicam que intervenções devem ser voltadas à motivação dos idosos para uma alimentação adequada, a qual previne tanto o baixo peso e a desnutrição quanto o sobrepeso e a obesidade. Talvez as instituições possam focalizar parte de sua prestação de cuidado a esse tipo de intervenção específica.

\section{DISCUSSÃO}

Estudos têm estimado a prevalência de desnutrição na população idosa institucionalizada entre 15 a $20 \%$, podendo atingir cifras de até $60 \%$ da amostra pesquisada, de acordo com o método e características da amostra e estudo analisado.

A desnutrição em idosos hospitalizados pode atuar negativamente no prognóstico clínicoe, consequentemente, aumentar o tempo de permanência hospitalar e a predisposição do idoso a complicações, tais como infecção e dificuldade na cicatrização, dentre outras.

Os valores de IMC e o Escore Total da Mini Avaliação Nutricional (MAN) puderam evidenciar um percentual que quanto maior a idade, maior o índice de desnutridos ou/e em risco de desnutrição neste grupo de idosos institucionalizados. O estado nutricional do idoso pode variar significativamente conforme o tipo de domicilio aonde reside o idoso. O estudo realizado com idosos residentes nesta instituição mostrou anciões com EN predominantemente desnutridos.

A Circunferência do Braço (CB) detectou o menor percentual de desnutrição. E não houve correlação quando se observou o comportamento de identificação dos desnutridos entre a $\mathrm{CP}$ e o IMC. Isso indica que a $\mathrm{CB}$ deve ser usada em conjunto com outros métodos, pois este não foi um método sensível na avaliação de desnutrição.

Além da questão do tipo de moradia, o gênero também pode influenciar sobremaneira os resultados de inquéritos antropométricos neste segmento etário. Em um estudo realizado em Santa Catarina em 2008 com idosos de ambos os gêneros, encontraram valores de IMC que categorizaram $45.5 \%$ da amostra em baixo-peso, $33.5 \%$ de indivíduos eutróficos, $7.8 \%$ de pré- obesos e $13.2 \%$ de obesos ${ }^{38}$. Encontraram resultados semelhantes no Piauí no mesmo ano, quando analisaram o EN pelo IMC, identificando que para homens idosos prevalecem a desnutrição $(70.3 \%)$ e eutrofia (18.5\%), ou seja, a maioria da amostra entre os baixos-pesos e eutróficos ${ }^{39}$.

Um estudo publicado afirmava que $57 \%$ da amostra de idosos estudados por esses autores definiam sua atual qualidade de vida como positiva. Os indivíduos mostravam-se insatisfeitos com sua qualidade de vida 
associando com mais problemas de saúde e sintomas depressivos $^{40}$.

Estes resultados obtidos confrontam os resultados de IMC encontrados em outros estudos nacionais desenvolvidos com idosos institucionalizados, como um estudo realizado no Distrito Federal, cuja amostra constituiu-se de 37 idosos institucionalizados, que indicou que houve a prevalência de eutrofia, mostrando-se presente em $47.9 \%$ dos idosos e em menor proporção idosos com baixo peso $(27.0 \%)$ e sobrepeso $(27.0 \%)$.

No entanto, em inquérito realizado em instituição mexicana de longa permanência para idosos, utilizando-se o protocolo de Mini-Avaliação Nutricional (MAN), encontraram prevalência de desnutrição de $6 \%$ em amostra de 55 residentes, mas $12 \%$ adicionais apresentavam risco de desnutrição, enquanto $44 \%$ da população estudada apresentava obesidade, valor superior a outros estudos ${ }^{41}$.

Ressalta-se que as intervenções em idosos devam possibilitar a manutenção da mobilidade, independência, nível de atividade, participação social e capacidade de responder adequadamente às demandas físicas e psicológicas da velhice ${ }^{42}$. De modo a poder atingir estes objetivos, é ímpar que se reconheçam quais fatores efetivamente se correlacionam e propiciam qualidade de vida aos anos de vida restantes. Poucos estudos têm, até o momento, se preocupado com a importância e o impacto do estado nutricional nas percepções de qualidade de vida dos idosos, institucionalizados ou não.

No caso de idosos demenciados e institucionalizados, os cuidadores são funcionários que, muitas vezes, não acompanham com exatidão a rotina dos pacientes devido à sobrecarga de trabalho e a troca rotineira de turnos, inviabilizando um tratamento e acompanhamento mais eficaz para estes pacientes. Sabe-se que a desnutrição do idoso, demenciado ou não, pode agravar doenças crônicodegenerativas já existentes, diminuindo sua independência.

Este estudo tem como limitações não ter analisado o impacto da presença de comorbidades, além de ser um estudo, que não permite inferências em âmbito do indivíduo idoso institucionalizado, em razão de restrições orçamentárias. Não foi possível estudar todas as duas ILPIs da cidade de Itabuna, apenas uma, pois ainda não houve autorização pela instituição, o que limitou a possibilidade de inferências estatísticas.

Apesar dos pontos positivos destacados, a impossibilidade de obter as informações necessárias para o preenchimento da MAN em idosos comprometidos cognitivamente e/ou desacompanhados é uma das limitações da sua aplicação, sendo essa a mesma desvantagem descrita na literatura. A imprecisão de informações fornecidas pelos cuidadores, sendo que esses frequentemente possuem elevada rotatividade.

As características da população estudada e a reduzida amostra, do mesmo modo, limitam a extrapolação dos achados desta pesquisa. Contudo, os resultados não são discrepantes de outros resultados para o Brasil e avançam no sentido de prover informações de interesses para os gestores das ILPIs e seus funcionários, devendo o cuidado com a alimentação do idoso ser parte central da rotina de cuidados.

Os resultados deste estudo, porém, reacendem a importância da discussão e melhor exploração dos dados encontrados em análises antropométricas, como o estado nutricional, e seu potencial impacto como um dos fatores a contribuir com percepções mais ou menos positivas ou negativas de qualidade de vida na velhice.

Diante do que foi discutido, verifica-se a necessidade de incluir o rastreio, avaliação e monitorização de idosos em estado de desnutrição ou risco de desnutrição no momento de admissão destes indivíduos em instituições de longa permanência, promover a educação nutricional no currículo destes cuidadores bem como conhecimentos sobre suporte nutricional e a criação de políticas específicas que incluíssem no mínimo, o rastreio nutricional periódico iria aumentar a capacidade de prevenção e sensibilização do mesmo.

\section{CONCLUSÃO}

Com o envelhecimento da população, enfrentar o desafio de estar preparado para o provimento de políticas específicas para este público se torna urgente, seja para o financiamento de estruturas de apoio, bem como para o monitoramento das suas atividades.

O país já tem um importante percentual de idosos, que será crescente nos próximos anos, demandando serviços públicos especializados que será reflexo do planejamento e das prioridades atuais das políticas públicas sociais.

Considerando os resultados observados no presente estudo, recomenda-se que a triagem nutricional e a classificação do estado nutricional de pacientes idosos façam parte da rotina de internação nas instituições geriátricas, pois a identificação precoce dos pacientes com risco nutricional e desnutrição permitirá a implantação de estratégias de intervenção adequadas à manutenção e/ou recuperação do estado nutricional. Considerando-se ainda que o protocolo de avaliação nutricional na maioria das instituições é realizado pelas equipes de enfermagem e cuidadores, sugere-se que essas recebam treinamento específico visando à aplicação da MAN. Essa atividade reforçará a atuação na atenção integral ao paciente, assim como contribuirá para o estabelecimento precoce e adequado de suporte nutricional pela equipe.

Conhecer o estado nutricional e as características associadas ao risco de desnutrição contribuem para a compreensão deste fenômeno e adequada avaliação e acompanhamento nutricional com detecção precoce dos indivíduos em risco de desnutrição e tratamento daqueles já desnutridos Tais medidas podem significar a prevenção de 
agravos relacionados à esta condição e melhora na qualidade de vida.

Através de uma intervenção nutricional individualizada, realizada depois de adequada avaliação do estado nutricional; e, quando necessário, das doenças existentes, será possível reverter, em grande número de casos, um quadro de desnutrição e, consequentemente, contribuir para proporcionar ao paciente idoso o restabelecimento de suas funções orgânicas.

\section{REFERÊNCIAS}

1. Organização Mundial da Saúde. Envelhecimento ativo: uma política de saúde. Brasília: OPAS; 2005.

2. Fazzio DM, Paz RC, Santos AB. Avaliação nutricional em idosos institucionalizados. Rev Divulg Cient Sena Aires. 2012;1(1):9-18.

3. Silva AKQ, Gusmão SC, Castro KR, Moreira RAN, Morais AHA. Perfil nutricional de idosos assistidos em instituições de longa permanência na cidade de Natal, RN. Geriatr Gerontol. 2010;4(1):27-35.

4. Duarte LMO. Processo de institucionalização do idoso e as territorialidades: espaço como lugar? Rev Nucleo Est Interdiscipl Envelhec. 2014;19(1):201-17.

5. Rissardo LK. Concepção e sentimentos de idosos institucionalizados sobre família. Rev Cienc Cuidado Saude. 2011;10(4):682-9.

6. Lopes BS. A idade é fator de risco de desnutrição em idosos institucionalizados. Rev Kairos Gerontol. 2015;18(1):353-60.

7. Instituto Brasileiro de Geografia e Estatística. Dados sobre população do Brasil, PNAD (pesquisa nacional por amostra de domicílios). Rio de Janeiro: IBGE; 2011.

8. Miranda GM, Mendes AC, Silva AA. O envelhecimento populacional brasileiro: desafios e consequências sociais atuais e futuras. Rev Bras Geriatr Gerontol. 2016;19(3).

9. Camarano AA, Kanso S. As instituições de longa permanência para idosos no Brasil. Rev Bras Estud Populacao. 2010;27(1).

10. Garcia CA, Moretto MC, Guariento ME. Estado nutricional e qualidade de vida em idosos. Rev Soc Bras Clin Med. 2016;14(1):52-6.

11. Beltrão KI, Camarano AA, Kanso S. Dinâmica populacional brasileira na virada do século XX. Rio de Janeiro: IPEA; 2004.

12. Bandeira L, Melo HP, Pinheiro LS. "Mulheres em dados: o que informa a PNAD/IBGE", 2008. Observatorio Brasil Igualdade Genero. 2010.

13. Kuchemann BA. Envelhecimento populacional, cuidados e cidadania: velhos dilemas e novos desafios. Rev Soc Est. 2012;27(1).

14. Campos MTFS, Monteiro JBR, Ornelas APRC. Fatores que afetam o consumo alimentar e a nutrição do idoso. Rev Nutr. 2000:157-65.

15. Esquenazi D, Guimarães MA. Aspectos fisiopatológicos no envelhecimento humano e quedas em idosos. Rev Hosp Univer Pedro Ernesto. 2012;13(2).
16. Chagas AM. Aspectos fisiológicos do envelhecimento e contribuição da odontologia na saúde do idoso. Rev Bras Odont. 2012;69(1):94-6.

17. Santelle, O, Lefevre AM C, Cervato AM. Alimentação institucionalizada e suas representações sociais entre moradores de instituições de longa permanência para idosos em São Paulo, Brasil. Cad Saude Publ. 2007;23(12).

18. Sousa VMC, Guariento MH .Avaliação do idoso desnutrido. Rev Bras Clin Med. 2009;7:46-9.

19. Stratton J, Lewy W, Cerqueira M, Schwartz RE, Abras, I. Cardio vascular responses to exercise effects of aging and exercise training in healthy men. Circulation. 1994;89:1648-55.

20. Shephard RJ. Envelhecimento, atividade física e saúde. São Paulo: Phorte; 2003.

21. De Vitta A. Atividade física e bem-estar na velhice. In: Néri AL, Freire SA, organizers. E por falar em boa velhice. Campinas: Papirus; 2000.

22. Gallahue DL, Ozmun JC. Compreendendo o desenvolvimento motor: bebês, crianças, adolescentes e adultos. 3rd ed. São Paulo: Phorte, 2005 .

23. Canineu PR, Bastos A. Transtorno cognitivo leve. In: Freitas EV, Py L, Néri AL, Cançado FAX, Gorzoni ML, Rocha SM, editors. Tratado de geriatria e gerontologia. Rio de Janeiro: Guanabara Koogan; 2002.

24. Cançado FAX, Horta ML. Envelhecimento cerebral. In: Freitas EV, Py L, Néri AL, Cançado FAX, Gorzoni ML, Rocha SM, editors. Tratado de geriatria e gerontologia. Rio de Janeiro: Guanabara Koogan; 2002.

25. Assis M. Aspectos sociais do envelhecimento. In: Saldanha AL, Caldas CP, editors. Saúde do idoso: a arte de cuidar. 2nd ed. Rio de Janeiro: Interciência; 2004

26. Teixeira MH. Aspectos psicológicos da velhice In: Saldanha AL, Caldas CP, editors. Saúde do idoso: a arte de cuidar. 2nd ed. Rio de Janeiro: Interciência; 2004.

27. Fidelix MS, Santana AF, Gomes JR. Prevalência de desnutrição hospitalar em idosos. Rev Assoc Bras Nutr. 2013;5(1).

28. Busnello FM. Aspectos nutricionais no processo do envelhecimento. São Paulo: Atheneu; 2007.

29. Silva JL, Marques AO, Leal MC, Alencar DL, Melo EM. Fatores associados à desnutrição em idosos institucionalizados. Rev Bras Geriatr Gerontol. 2014;18(2):443-51.

30. Souza KT. Baixo peso e a dependência funcional em idosos institucionalizados. Rev Cienc Saude Colet. 2014;19(8):3513-20.

31. Cardozo NR. Estado nutricional de idosos atendidos por unidades de saúde da família na cidade de Pelotas-RS. J Soc Bras Nutr Enteral Parenteral. 2017;32:94-8.

32. Padilha LM. Avaliação do nível assistencial nutricional em pacientes adultos internados no hospital de clínicas de Porto Alegre. Rev Hosp Clin Porto Alegre. 2008;28(3):158-61.

33. BRASIL. Portaria ${ }^{\circ} 810$ aprova as normas e padrões para o funcionamento as casas de repouso, clínicas geriátricas e outras instituições destinadas ao atendimento de idosos. Brasília; 1989.

34. Sociedade Brasileira de Geriatria e Gerontologia. Instituições de longa permanência para idosos: manual de funcionamento. São Paulo: SBGG; 2004. 
35. Born T, Boechat NS. A qualidade dos cuidados aos idosos institucionalizados. In: Freitas EV. Tratado de geriatria e gerontologia. Rio de Janeiro: Guanabara Koogan; 2006.

36. Camargos MC, Carvalho GW, Nascimento DI, Machado CJ. Aspectos relacionados à alimentação em instituições de longa permanência para idosos em Minas Gerais. Cad Saude Colet. 2015;23(1):38-43.

37. Santelle $O, L$ efevre AM C, Cervato AM. Alimentação institucionalizada e suas representações sociais entre moradores de instituições de longa permanência para idosos em São Paulo, Brasil. Cad Saude Publ. 2007;23(12).

38. Rauen MS, Moreira EAM, Calvo MCM, Lobo AS. Avaliação do estado nutricional de idosos institucionalizados. Rev Nutr. 2008;21(3):303-10.

39. Melo MTS. Avaliação do estado nutricional de idosos residentes em Instituições de longa permanência em Teresina [dissertation]. Teresina (PI): Universidade Federal do Piauí; 2008.

40. Xavier FMF. Elderly people's definition of quality of life. Rev Bras Psiquiatr. 2003;25(1):31-9.

41. Álamo AC. Protocolo de valoración, seguimiento y actuación nutricional en un residencial para personas mayores. Nutr Hospitalaria. 2008;23(2):100-4.

42. Chachamovich E. Qualidade de vida em idosos: desenvolvimento e aplicação do módulo WHOQOL-Old e teste do desempenho do instrumento WHOQOL-bref em uma amostra de idosos brasileiros [dissertation]. Porto Alegre (RS): Universidade Federal do Rio Grande do Sul; 2005. 